\section{The cancer epigenome of mice and men}

Genetically engineered mouse models (GEMMs) of human disease rarely take into account epigenetic alterations, which contribute to many human cancers. Now, Stephen Tapscott and colleagues compare genome-wide patterns of cancer-specific DNA methylation in human patients and three GEMMs of medulloblastoma (Epigenetics 8, 1254-1260, 2013). Using two independent methods to measure CpG methylation, they find, in contrast to the hypermethylation patterns previously observed in patients with medulloblastoma, that GEMMs had only modest increase in CpG methylation of gene promoters relative to wildtype controls. Whereas a human medulloblastoma tumor sample showed $>60 \%$ increase in methylation at 121 loci, consistent with the authors' previous work, there were only 0-16 such loci in the GEMMs. They further extend these findings to mouse models of Burkitt lymphoma and breast cancer, which showed similar results. The authors confirmed, using immortalized mouse fibroblast cell lines, that the machinery necessary for promoter hypermethylation is present in mouse cells but is not activated in oncogene-driven GEMMs of cancer. The authors suggest that these data will be useful in developing more precise GEMMs for preclinical studies, which are especially crucial in identifying drugs that target the epigenome.

\section{Isogenic iPSC-derived models of disease}

The use of patient-derived induced pluripotent stem cells (iPSCs) to study genetic mechanisms of disease has been limited by the confounding effect of genetic background. Now, Rudolf Jaenisch, Rajesh Ambasudhan, Stuart Lipton and colleagues report the use of isogenic iPSC-derived models of a dominant familial form of Parkinson's disease to study disease mechanisms (Cell 155, 1351-1364, 2013). The authors used previously generated human iPSCs from an individual with the coding mutation A53T in SNCA, which encodes a-synuclein, and an isogenic line in which this mutation was corrected in the endogenous $\alpha$-synuclein locus using zinc-finger nuclease-mediated genome editing. The authors differentiated these cells into A9 dopaminergic neurons, the cell type affected in Parkinson's disease. They found that the A53T alteration caused increased amounts of $\alpha$-synuclein protein aggregate, deficits in mitochondrial respiration and increased susceptibility to mitochondrial toxins. The authors determined that cells with the A53T alteration had increased reactive oxygen species and nitric oxide production, which disrupted a MEF2CPGC1 $\alpha$-mediated neuroprotective pathway. The authors suggest that the MEF2C-PGC1 a pathway may be a promising target for the development of therapeutics to treat Parkinson's disease.

\section{OXA resistance from QTL to crystal structure}

Tim Anderson and colleagues characterize the genetic and molecular basis of oxamniquine (OXA) resistance in Schistosoma mansoni, a trematode parasite (Science doi:10.1126/science.1243106, 21 November 2013). They performed linkage mapping in the second-generation progeny of a cross between a sensitive and a resistant parasite, identifying a single quantitative trait locus (QTL) that included 16 candidate genes. They used a biochemical complementation assay to test which of the candidate genes produced a protein that is able to convert OXA, a pro-drug, to an active form of the drug as present in sensitive but not resistant parasites. They found that Smp_089320 activated OXA in extracts from resistant

Written by Orli Bahcall, Brooke LaFlamme, Emily Niemitz \& Kyle Vogan parasites and confirmed its role in OXA resistance by showing that RNA interference knockdown of Smp_089320 in drug-sensitive parasites resulted in an increase in resistance. They found that the Smp_089320 protein in sensitive but not resistant strains showed sulfonation activity, identifying a mechanism for the drug in which it acts as a sulfotransferase that activates OXA. Finally, the authors determined the crystal structure of Smp_089320 protein from sensitive parasites with OXA bound and suggest that the mechanism of resistance involves disruption of the drug-protein interaction in resistant strains. Comparative and phylogenetic analysis with other schistosomes also suggests the basis for the species specificity in OXA drug action.

$O B$

\section{ELABELA, a peptide hormone for heart development}

Bruno Reversade and colleagues identify a highly conserved gene encoding a peptide hormone required for cardiac development in zebrafish (Dev. Cell doi:10.1016/j.devcel.2013.11.002, 5 December 2013). The gene, ELABELA, is found on human chromosome 4 and was previously identified as a noncoding RNA. However, the authors found that ELA contains a conserved ORF predicted to express a 54-residue secreted protein. The mature form of ELA is only 32 amino acids, with the last 13 residues being nearly invariant among all sequenced vertebrates. The authors used an allelic series of zebrafish ela mutants to show that Ela deficiency leads to severe defects in cardiac morphogenesis and often results in the complete absence of a heart. The ela mutant phenotype is similar to that of Apelin receptor (Aplnr) mutants, and both Ela and Aplnr are expressed before gastrulation. In contrast, the mutant for the currently accepted Aplnr ligand, Apelin, does not recapitulate the Aplnr phenotype, and Apelin is expressed only later in development. These results indicate that ELABELA is the Aplnr ligand responsible for cardiac development. ELA is expressed in human embryonic stem cells and adult human prostate and kidney. The authors suggest that ELA may have cardioprotective and/or vasodilatory properties in humans, as does Apelin.

$B L$

\section{ARMC5 and adrenal hyperplasia}

Jérôme Bertherat and colleagues (N. Engl. J. Med. 369, 2105-2114, 2013) identify biallelic inactivation of ARMC5 as a recurrent driver event in corticotropin-independent macronodular adrenal hyperplasia, a rare subtype of Cushing syndrome characterized by multiple, bilateral adrenocortical tumors. Using SNP arrays, the authors analyzed 34 tumor samples from 26 affected individuals and identified a region at $16 \mathrm{p} 11$ showing loss of heterozygosity in multiple samples. In parallel, they performed whole-genome sequencing of five tumors and paired normal samples and identified three tumors with somatic alterations in $A R M C 5$, a gene of unknown function mapping to the $16 \mathrm{p} 11$ candidate region. Further sequencing of tumor DNA from 33 affected individuals identified 18 samples with ARMC5 alterations, including several nonsense and frameshift mutations. Analysis of constitutive DNA samples from 14 of these individuals identified germline ARMC5 alterations in all cases. More detailed analyses showed that the tumors acquired a second somatic alteration leading to biallelic inactivation of ARMC5, consistent with a two-hit tumor suppressor model. Although further studies are needed to elucidate the pathophysiological mechanisms, ARMC5-mutated tumors exhibit a distinct gene expression signature that may provide insights into deregulated pathways. $K V$ 